\title{
Estimates for the Chebyshev Function $\psi(x)-\theta(x)$
}

\section{By N. Costa Pereira*}

Abstract. A simple approximation for the difference $\psi(x)-\theta(x)$ is established by elementary methods. This approximation is used to obtain several estimates for $\psi(x)-\theta(x)$ which are sharper than those previously given in the literature.

Let $\psi$ and $\theta$ be defined, as usual, by

$$
\psi(x)=\sum_{p^{\alpha} \leqslant x} \log p ; \quad \theta(x)=\sum_{p \leqslant x} \log p
$$

with $x>0, p$ prime and $\alpha$ a positive integer. In this paper we show that it is possible to approximate the difference $\psi-\theta$ in terms of $\psi$ in quite a simple form. As consequences we deduce some estimates for $\psi-\theta$ which improve those given in [3], [4], and [5].

THEOREM 1. For every $x>0$ we have

$$
\begin{aligned}
& \psi(x)-\theta(x) \leqslant \psi\left(x^{1 / 2}\right)+\psi\left(x^{1 / 3}\right)+\psi\left(x^{1 / 5}\right), \\
& \psi(x)-\theta(x) \geqslant \psi\left(x^{1 / 2}\right)+\psi\left(x^{1 / 3}\right)+\psi\left(x^{1 / 7}\right) .
\end{aligned}
$$

Proof. From the well-known identity

$$
\psi(x)=\sum_{k \geqslant 1} \theta\left(x^{1 / k}\right),
$$

we deduce

$$
\psi\left(x^{1 / 2}\right)=\sum_{k \geqslant 1} \theta\left(x^{1 / 2 k}\right)
$$

Substituting (4) in (3) we get

$$
\psi(x)=\theta(x)+\psi\left(x^{1 / 2}\right)+\sum_{k \geqslant 1} \theta\left(x^{1 / 2 k+1}\right)
$$

or

$$
\begin{aligned}
\psi(x)-\theta(x)= & \psi\left(x^{1 / 2}\right)+\sum_{k \geqslant 1} \theta\left(x^{1 / 6 k-3}\right) \\
& +\sum_{k \geqslant 1} \theta\left(x^{1 / 6 k-1}\right)+\sum_{k \geqslant 1} \theta\left(x^{1 / 6 k+1}\right) .
\end{aligned}
$$

Received July 8, 1981; revised March 1, 1984.

1980 Mathematics Subject Classification. Primary 10A20; Secondary $10 \mathrm{H} 15$.

* Supported in part by a grant from Secretaria de Estado da Cultura, Lisbon, Portugal. 
From (3) we can still deduce

$$
\psi\left(x^{1 / 3}\right)=\sum_{k \geqslant 1} \theta\left(x^{1 / 3 k}\right)=\sum_{k \geqslant 1} \theta\left(x^{1 / 6 k-3}\right)+\sum_{k \geqslant 1} \theta\left(x^{1 / 6 k}\right)
$$

and (5) is transformed into

$$
\begin{aligned}
\psi(x)-\theta(x)= & \psi\left(x^{1 / 2}\right)+\psi\left(x^{1 / 3}\right)+\sum_{k \geqslant 1} \theta\left(x^{1 / 6 k-1}\right) \\
& -\sum_{k \geqslant 1} \theta\left(x^{1 / 6 k}\right)+\sum_{k \geqslant 1} \theta\left(x^{1 / 6 k+1}\right) .
\end{aligned}
$$

Since $\theta$ is an increasing function, from (6) we get the inequalities

$$
\begin{aligned}
\psi(x)-\theta(x) & \leqslant \psi\left(x^{1 / 2}\right)+\psi\left(x^{1 / 3}\right)+\sum_{k \geqslant 1} \theta\left(x^{1 / 6 k-1}\right) \\
& \leqslant \psi\left(x^{1 / 2}\right)+\psi\left(x^{1 / 3}\right)+\sum_{k \geqslant 1} \theta\left(x^{1 / 5 k}\right)
\end{aligned}
$$

and

$$
\begin{aligned}
\psi(x)-\theta(x) & \geqslant \psi\left(x^{1 / 2}\right)+\psi\left(x^{1 / 3}\right)+\sum_{k \geqslant 1} \theta\left(x^{1 / 6 k+1}\right) \\
& \geqslant \psi\left(x^{1 / 2}\right)+\psi\left(x^{1 / 3}\right)+\sum_{k \geqslant 1} \theta\left(x^{1 / 7 k}\right) .
\end{aligned}
$$

Using (3) again, we obtain

$$
\psi\left(x^{1 / 5}\right)=\sum_{k \geqslant 1} \theta\left(x^{1 / 5 k}\right) ; \quad \psi\left(x^{1 / 7}\right)=\sum_{k \geqslant 1} \theta\left(x^{1 / 7 k}\right) .
$$

Substituting these identities respectively in (7) and (8), we get (1) and (2), which proves the theorem.

Other estimates for $\psi-\theta$ could be obtained with the methods used in the preceding proof. As an example, observing that

$$
\begin{aligned}
& \sum_{k \geqslant 1} \theta\left(x^{1 / 6 k-1}\right)+\sum_{k \geqslant 1} \theta\left(x^{1 / 6 k+1}\right) \\
& \geqslant \sum_{k \geqslant 1} \theta\left(x^{1 / 10 k-5}\right)+\sum_{k \geqslant 1} \theta\left(x^{1 / 10 k}\right)=\psi\left(x^{1 / 5}\right),
\end{aligned}
$$

we get from (6),

$$
\psi(x)-\theta(x) \geqslant \psi\left(x^{1 / 2}\right)+\psi\left(x^{1 / 3}\right)+\psi\left(x^{1 / 5}\right)-\psi\left(x^{1 / 6}\right),
$$

which is sharper than (2) for large values of $x$. Combining this inequality with (1), we have

$$
\psi(x)-\theta(x)=\psi\left(x^{1 / 2}\right)+\psi\left(x^{1 / 3}\right)+\psi\left(x^{1 / 5}\right)-\xi \psi\left(x^{1 / 6}\right)
$$

with $0 \leqslant \xi \leqslant 1$.

The inequalities given by Theorem 1 are, however, sharp enough for our present purposes. In order to apply Theorem 1, we have first constructed a table of $\psi(x)-\theta(x)$ in the range $0<x \leqslant 3 \cdot 10^{6}$ with an accuracy of five decimals (Table I). Since $\psi(x)-\theta(x)$ is a step function that only jumps where $x=p^{\alpha}$ with $p$ prime and $\alpha$ an integer greater than one, we only need to tabulate $\psi(x)-\theta(x)$ for those values of $x$.

We will also use two simple lemmas. 
LEMMA 1. If an interval $I$ is the union of a finite collection of intervals $I_{k}=\left(m_{k}, n_{k}\right)$, and there are positive constants $a_{k}, b_{k}, c_{k}, L^{+}$and $a$ constant $\varepsilon \geqslant 0$, such that $a_{k}>1+\varepsilon$ and

$$
\begin{gathered}
\psi\left(x^{1 / 2}\right)<a_{k} x^{1 / 2} ; \quad \psi\left(x^{1 / 3}\right)<b_{k} x^{1 / 3}, \\
\psi\left(x^{1 / 5}\right)<c_{k} x^{1 / 5} \quad \text { for } x \in I_{k}, \\
m_{k} \geqslant\left(4 c_{k} / 5\left(a_{k}-1-\varepsilon\right)\right)^{10 / 3}, \\
L^{+} \geqslant \sup _{k}\left\{\left(a_{k}-1-\varepsilon\right) n_{k}^{1 / 6}+b_{k}+c_{k} n_{k}^{-2 / 15}\right\},
\end{gathered}
$$

we have

$$
\psi(x)-\theta(x)<(1+\varepsilon) x^{1 / 2}+L^{+} x^{1 / 3} \text { for } x \in I .
$$

Proof. If $x \in I_{k}$, from Theorem 1 and (9) it follows that

$$
\psi(x)-\theta(x)<a_{k} x^{1 / 2}+b_{k} x^{1 / 3}+c_{k} x^{1 / 5} \quad \text { if } x \in I_{k},
$$

or,

$$
\begin{aligned}
& \psi(x)-\theta(x)<(1+\varepsilon) x^{1 / 2} \\
& \left(\left(a_{k}-1-\varepsilon\right) x^{1 / 6}+b_{k}+c_{k} x^{-2 / 15}\right) x^{1 / 3} \quad \text { if } x \in I_{k} .
\end{aligned}
$$

The coefficient of $x^{1 / 3}$ in this last expression increases monotonically in $I_{k}$ provided that

$$
x \geqslant\left(4 c_{k} / 5\left(a_{k}-1-\varepsilon\right)\right)^{10 / 3} \text { for } x \in I_{k}
$$

and this condition is implied by (10). Thus, we get

$$
\psi(x)-\theta(x)<(1+\varepsilon) x^{1 / 2}+\left(\left(a_{k}-1-\varepsilon\right) n_{k}^{1 / 6}+b_{k}+c_{k} n_{k}^{-2 / 15}\right) x^{1 / 3}
$$

if $x \in I_{k}$, and the lemma follows from (11).

LEMMA 2. If an interval $J$ is the union of a finite collection of intervals $J_{k}=\left(m_{k}, n_{k}\right)$, and there are positive constants $a_{k}^{\prime}, b_{k}^{\prime}, d_{k}, L^{-}$and a constant $\varepsilon \geqslant 0$ such that $a_{k}^{\prime}<1$ $-\varepsilon$ and

$$
\begin{gathered}
\psi\left(x^{1 / 2}\right)>a_{k}^{\prime} x^{1 / 2} ; \quad \psi\left(x^{1 / 3}\right)>b_{k}^{\prime} x^{1 / 3}, \\
\psi\left(x^{1 / 7}\right)>d_{k} x^{1 / 7} \quad \text { for } x \in J_{k}, \\
L^{-} \leqslant \inf _{k}\left\{\left(a_{k}^{\prime}-1+\varepsilon\right) n_{k}^{1 / 6}+b_{k}^{\prime}+d_{k} n_{k}^{-4 / 21}\right\},
\end{gathered}
$$

we have

$$
\psi(x)-\theta(x)>(1-\varepsilon) x^{1 / 2}+L^{-} x^{1 / 3} \quad \text { for } x \in J .
$$

Proof. If $x \in J_{k}$, from Theorem 1 and (12), we have

$$
\psi(x)-\theta(x)>a_{k}^{\prime} x^{1 / 2}+b_{k}^{\prime} x^{1 / 3}+d_{k} x^{1 / 7}
$$

or

$$
\begin{aligned}
\psi(x)-\theta(x)> & (1-\varepsilon) x^{1 / 2} \\
& +\left(\left(a_{k}^{\prime}-1+\varepsilon\right) x^{1 / 6}+b_{k}^{\prime}+d_{k} x^{-4 / 21}\right) x^{1 / 3} \quad \text { if } x \in J_{k} .
\end{aligned}
$$


The coefficient of $x^{1 / 3}$ in this last expression clearly decreases when $x$ increases and we get, if $x \in J_{k}$,

$$
\psi(x)-\theta(x)>(1-\varepsilon) x^{1 / 2}+\left(\left(a_{k}^{\prime}-1+\varepsilon\right) n_{k}^{1 / 6}+b_{k}^{\prime}+d_{k} n_{k}^{-4 / 21}\right) x^{1 / 3} .
$$

Now the lemma follows from (13).

It is now possible to show the following

THEOREM 2. We have

$$
\begin{array}{ll}
\psi(x)-\theta(x)<\sqrt{x}+\frac{4}{3} \sqrt[3]{x} & \text { if } 0<x \leqslant 10^{8}, \\
\psi(x)-\theta(x)>\sqrt{x}+\frac{2}{3} \sqrt[3]{x} & \text { if } 2187 \leqslant x \leqslant 10^{8} .
\end{array}
$$

Proof. Inequalities (14) and (15) can be verified directly from our Table I for $x \leqslant 3 \cdot 10^{6}$. To prove (14) in this range we start with $x>0$ and we get

$$
\sqrt{x}+\frac{4}{3} \sqrt[3]{x}>0=\lim _{t \rightarrow 4^{-}} \psi(t)-\theta(t)=\psi(x)-\theta(x) \text { if } 0<x<4 .
$$

Next, we take $x \geqslant 4$ and Table I gives

$$
\sqrt{x}+\frac{4}{3} \sqrt[3]{x}>4.116>\lim _{t \rightarrow 25^{-}} \psi(t)-\theta(t) \geqslant \psi(x)-\theta(x) \quad \text { if } 4 \leqslant x<25 .
$$

Continuing in the same way and noticing that $\psi\left(3 \cdot 10^{6}\right)-\theta\left(3 \cdot 10^{6}\right)$ is given by the last entry in the table, we establish (14) for $x \leqslant 3 \cdot 10^{6}$.

To verify (15) in the range $2187 \leqslant x \leqslant 3 \cdot 10^{6}$ we start at the end of Table I. Taking $x \leqslant 3 \cdot 10^{6}$, we have

$$
\sqrt{x}+\frac{2}{3} \sqrt[3]{x}<1828.201<\psi\left(x_{1}\right)-\theta\left(x_{1}\right)
$$

with $x_{1}=2765569$, and this gives (15) for $x_{1} \leqslant x \leqslant 3 \cdot 10^{6}$. Next, taking $x<x_{1}$ we have

$$
\sqrt{x}+\frac{2}{3} \sqrt[3]{x}<1756.578<\psi\left(x_{2}\right)-\theta\left(x_{2}\right)
$$

with $x_{2}=2571353$, and this gives (15) for $x_{2} \leqslant x<x_{1}$. This process can be continued down to $x=2187$ and (15) is established for $x \leqslant 3 \cdot 10^{6}$.

If $3 \cdot 10^{6}<x \leqslant 10^{8}$ we have

$$
1732<x^{1 / 2} \leqslant 10^{4} ; \quad 144<x^{1 / 3}<465 ; \quad 19<x^{1 / 5}<40 ; \quad 8<x^{1 / 7}<14 .
$$

From the Appel and Rosser [1] table of $\theta(x)$, Lehmer's [2] table of primes, and Table I, it follows that we can apply Lemma 2 to the interval $J=J_{1}=\left(3 \cdot 10^{6}, 10^{8}\right]$ with

$$
\varepsilon=0 ; \quad L^{-}=2 / 3 ; \quad a_{1}^{\prime}=0.98708 ; \quad b_{1}^{\prime}=0.94842 ; \quad d_{1}=0.71200,
$$

and this proves (15).

To prove (14) in the interval $I=\left(3 \cdot 10^{6}, 10^{8}\right]$, we consider the intervals $I_{1}$ $=\left(3 \cdot 10^{6}, 83.5 \cdot 10^{6}\right]$ and $I_{2}=\left(83.5 \cdot 10^{6}, 10^{8}\right]$. Using the same tables it is possible to apply Lemma 2 with

$$
\begin{gathered}
\varepsilon=0 ; \quad L^{+}=4 / 3 ; \quad a_{1}=1.00990 ; \quad a_{2}=1.00463 ; \\
b_{1}=b_{2}=1.03591 ; \quad c_{1}=c_{2}=1.02938,
\end{gathered}
$$

and this establishes (14). 
From Theorem 2, we can deduce the following estimates for $\psi(x)$ in the range $0<x \leqslant 10^{8}$ :

THEOREM 3. We have

$$
\begin{gathered}
\psi(x)<x+0.656 \sqrt{x}+\frac{4}{3} \sqrt[3]{x} \quad \text { if } 0<x \leqslant 10^{8}, \\
\psi(x)>x-0.833 \sqrt{x}+\frac{2}{3} \sqrt[3]{x} \\
\text { if } 1427 \leqslant x \leqslant 3298,3299 \leqslant x \leqslant 19371 \text { or } 19373 \leqslant x \leqslant 10^{8} .
\end{gathered}
$$

Proof. From the Appel and Rosser [1] tables of $\theta(x)$ and $(x-\theta(x)) / \sqrt{x}$, we have

$$
\frac{x-\theta(x)}{\sqrt{x}}>0.344 \text { if } 0<x \leqslant 10^{8},
$$

and

$$
\frac{x-\theta(x)}{\sqrt{x}}<1.833 \text { if } 19801 \leqslant x \leqslant 10^{8} .
$$

These inequalities, together with (14) and (15), prove (16) for $0<x \leqslant 10^{8}$ and (17) for $19801 \leqslant x \leqslant 10^{8}$. Directly from [1], [2] and Table I, we can verify that (17) still holds when $1427 \leqslant x \leqslant 3298,3299 \leqslant x \leqslant 19371$ and $19373 \leqslant x<19801$.

We have applied Theorem 3 together with the tables [1], [2] and Table I to get some close estimates for $\psi(x)$ in the range $0<x \leqslant 10^{8}$. In Table II, we have listed triplets $\left(n, \lambda^{-}, \lambda^{+}\right)$such that

$$
\lambda^{-} x<\psi(x)<\lambda^{+} x \text { if } n \leqslant x \leqslant 10^{8} .
$$

To evaluate the lower bounds $\lambda^{-}$, we observe that

$$
\psi(x) / x>\psi\left(n_{k}\right) / n_{k+1} \text { for } n_{k} \leqslant x<n_{k+1},
$$

where $n_{k}$ and $n_{k+1}$ are two consecutive prime powers. Then, if $n_{k}$ is the largest prime power (not exceeding $10^{8}$ ) such that $\psi\left(n_{k-1}\right) / n_{k}<\lambda^{-}$, it follows that $\psi(x)>\lambda^{-} x$ for $n_{k} \leqslant x \leqslant 10^{8}$; it also follows that this inequality fails immediately below $n_{k}$.

Now, in the range $10^{8} \leqslant x \leqslant 10^{16}$, we can get better estimates for $\psi(x)-\theta(x)$ than those given by (14) and (15).

THEOREM 4. We have

$$
\begin{array}{ll}
\psi(x)-\theta(x)<\sqrt{x}+\frac{6}{5} \sqrt[3]{x} & \text { if } 10^{8} \leqslant x \leqslant 10^{16}, \\
\psi(x)-\theta(x)>\sqrt{x}+\frac{6}{7} \sqrt[3]{x} & \text { if } 10^{8} \leqslant x \leqslant 10^{16} .
\end{array}
$$

Proof. To prove (18) we divide $I=\left[10^{8}, 10^{16}\right]$ into intervals $I_{k}=\left[m_{k}, n_{k}\right)$ for $k=1, \ldots, 8$ and $I_{9}=\left[m_{9}, 10^{16}\right]$. Using the tables [1], [2] together with (16) and Tables I and II, it is now easy to verify that we can apply Lemma 1 with $\varepsilon=0$, $L^{+}=6 / 5$, and the following constants $m_{k}, n_{k}, a_{k}, b_{k}, c_{k}$. 


\begin{tabular}{l|l|l|l|l|l}
$k$ & \multicolumn{1}{|c|}{$m_{k}$} & \multicolumn{1}{c|}{$n_{k}$} & $a_{k}$ & $b_{k}$ & $c_{k}$ \\
\hline 1 & $10^{8}$ & $10273^{2}$ & 1.00347 & 1.02117 & 0.90967 \\
\hline 2 & $10273^{2}$ & $41^{5}$ & 1.00517 & 1.00913 & 0.90967 \\
\hline 3 & $41^{5}$ & $661^{3}$ & 1.00421 & 1.01130 & 1.01604 \\
\hline 4 & $661^{3}$ & $24103^{2}$ & 1.00347 & 1.01802 & 1.01604 \\
\hline 5 & $24103^{2}$ & $839^{3}$ & 1.00458 & 0.99829 & 0.94445 \\
\hline 6 & $839^{3}$ & $10^{10}$ & 1.00297 & 1.01364 & 1.02871 \\
\hline 7 & $10^{10}$ & $16 \cdot 10^{11}$ & 1.00153 & 1.00990 & 1.03883 \\
\hline 8 & $16 \cdot 10^{11}$ & $2 \cdot 10^{15}$ & 1.00049 & 1.00990 & 1.02728 \\
\hline 9 & $2 \cdot 10^{15}$ & $10^{16}$ & 1.00037 & 1.00990 & 1.01364 \\
\hline
\end{tabular}

Similarly, to prove (19), we divide $J=\left[10^{8}, 10^{16}\right]$ into intervals $J_{k}=\left[m_{k}, n_{k}\right.$ ) for $k=1, \ldots, 7$ and $J_{8}=\left[m_{8}, 10^{16}\right]$. Again using [1], [2] together with (17) and Tables I and II, we can apply Lemma 2 with $\varepsilon=0, L^{-}=6 / 7$ and the following constants $m_{k}, n_{k}, a_{k}^{\prime}, b_{k}^{\prime}, d_{k}$.

\begin{tabular}{c|l|l|c|c|c}
$k$ & \multicolumn{1}{|c|}{$m_{k}$} & \multicolumn{1}{|c|}{$n_{k}$} & $a_{k}^{\prime}$ & $b_{k}^{\prime}$ & $d_{k}$ \\
\hline 1 & $10^{8}$ & $11801^{2}$ & 0.99343 & 0.99189 & 0.87827 \\
\hline 2 & $11801^{2}$ & $18500^{2}$ & 0.99486 & 0.97494 & 0.79967 \\
\hline 3 & $18500^{2}$ & $20000^{2}$ & 0.99486 & 0.97870 & 0.79631 \\
\hline 4 & $20000^{2}$ & $53000^{2}$ & 0.99643 & 0.97870 & 0.79341 \\
\hline 5 & $53000^{2}$ & $180000^{2}$ & 0.99770 & 0.97870 & 0.83763 \\
\hline 6 & $180000^{2}$ & $121 \cdot 10^{10}$ & 0.99870 & 0.98828 & 0.88117 \\
\hline 7 & $121 \cdot 10^{10}$ & $15 \cdot 10^{14}$ & 0.99960 & 0.99343 & 0.90602 \\
\hline 8 & $15 \cdot 10^{14}$ & $10^{16}$ & 0.99987 & 0.99851 & 0.94842 \\
\hline
\end{tabular}

With the aid of a computer, it can be easily verified that inequalities (18) and (19) still hold, respectively, for $8236167 \leqslant x \leqslant 10^{8}$ and $2036329 \leqslant x \leqslant 10^{8}$ but fail immediately below these bounds. We conclude

$$
\psi(x)-\theta(x)<\sqrt{x}+\frac{6}{5} \sqrt[3]{x} \quad \text { if } 8236167 \leqslant x \leqslant 10^{16}
$$

and

$$
\psi(x)-\theta(x)>\sqrt{x}+\frac{6}{7} \sqrt[3]{x} \quad \text { if } 2036329 \leqslant x \leqslant 10^{16}
$$

The results we have proved so far are strictly elementary. However, in order to estimate $\psi(x)-\theta(x)$ for $x>10^{16}$, we need the following bounds for $\psi$ which were recently deduced by Schoenfeld [5], using powerful analytical methods. 


$$
\begin{array}{ll}
|\psi(x)-x|<0.00119721 x & \text { if } 10^{8} \leqslant x<e^{18.43} \\
|\psi(x)-x|<0.0011930 x & \text { if } e^{18.43} \leqslant x<e^{18.44} \\
|\psi(x)-x|<0.0011885 x & \text { if } e^{18.44} \leqslant x<e^{18.45} \\
|\psi(x)-x|<0.0011839 x & \text { if } e^{18.45} \leqslant x<e^{18.5} \\
|\psi(x)-x|<0.0011615 x & \text { if } e^{18.5} \leqslant x<e^{18.7} \\
|\psi(x)-x|<0.0010765 x & \text { if } e^{18.7} \leqslant x<e^{19} \\
|\psi(x)-x|<0.00096161 x & \text { if } x \geqslant e^{19}
\end{array}
$$

We can now prove the following

\section{THEOREM 5. We have}

$$
\begin{array}{ll}
\psi(x)-\theta(x)<1.001 \sqrt{x}+1.1 \sqrt[3]{x} & \text { if } x \geqslant 10^{16}, \\
\psi(x)-\theta(x)<1.001 \sqrt{x}+\sqrt[3]{x} & \text { if } x \geqslant e^{38} \\
\psi(x)-\theta(x)>0.999 \sqrt{x}+0.9 \sqrt[3]{x} & \text { if } x \geqslant 10^{16} \\
\psi(x)-\theta(x)>0.999 \sqrt{x}+\sqrt[3]{x} & \text { if } x \geqslant e^{38}
\end{array}
$$

Proof. To prove (29) we divide $I=\left[10^{16}, e^{38}\right)$ into intervals $I_{1}=\left[10^{16}, e^{36.88}\right)$, $I_{2}=\left[e^{36.88}, e^{37}\right), I_{3}=\left[e^{37}, e^{38}\right)$. Using [1], [2] and Tables I and II together with estimates (22) to (27), we see that it is possible to apply Lemma 1 with $\varepsilon=0.001$, $L^{+}=1.1$, and

$$
\begin{gathered}
a_{1}=1.00119721, \quad a_{2}=1.0011885, \quad a_{3}=1.0011615, \\
b_{1}=b_{2}=1.00052, \quad b_{3}=1.00121, \\
c_{1}=1.00450, \quad c_{2}=c_{3}=1.01364
\end{gathered}
$$

Now, if $x \geqslant e^{38}$, it follows from (1), (22) to (28) and Table II that

$$
\begin{aligned}
\psi(x)-\theta(x) & <1.001 x^{1 / 2}+\left(-0.00003839 x^{1 / 6}+1.00115+1.00990 x^{-2 / 15}\right) x^{1 / 3} \\
& <1.001 x^{1 / 2}+x^{1 / 3}
\end{aligned}
$$

and this proves (29) and (30).

To prove (31), we divide $J=\left[10^{16}, e^{38}\right)$ into intervals $J_{1}=\left[10^{16}, e^{37}\right), J_{2}=$ $\left[e^{37}, e^{38}\right)$. Using inequalities (22) to (27) together with Table II, it is easily seen that we can apply Lemma 2 with $\varepsilon=0.001, L^{-}=0.9$, and

$$
a_{1}^{\prime}=0.99880279, \quad a_{2}^{\prime}=0.9988385, \quad b_{1}^{\prime}=b_{2}^{\prime}=0.99870, \quad d_{1}=d_{2}=0.94842 \text {. }
$$

Finally, if $x \geqslant e^{38}$, it follows from (2), (22) to (28) and Table II that

$$
\begin{aligned}
\psi(x)-\theta(x) & >0.999 x^{1 / 2}+\left(0.00003839 x^{1 / 6}+0.99880\right) x^{1 / 3} \\
& >0.999 x^{1 / 2}+x^{1 / 3},
\end{aligned}
$$

and this proves (31) and (32). 
TABLE I

\begin{tabular}{|c|c|c|c|c|c|c|c|}
\hline & $\psi(x)-\theta(x)$ & $\approx$ & $\Psi(x)-\theta(x)$ & $\underline{x}$ & $\langle\psi(x)-\theta(x)$ & & $\Psi(x)-\theta(x)$ \\
\hline 4 & 0.69315 & 10201 & 122.02043 & 80089 & 340.44853 & 292681 & 606.67063 \\
\hline 8 & & 10609 & 126.65516 & 83521 & 8174 & & 0750 \\
\hline 9 & 3491 & 49 & & 49 & 32 & & \\
\hline 16 & 805 & 11881 & & 49 & 76 & & \\
\hline 25 & .78749 & 12167 & I & 96721 & 42855 & & \\
\hline 27 & & 12769 & 222 & 97969 & 7476 & 761 & \\
\hline 32 & & 14641 & 012 & 100489 & & & \\
\hline 49 & & 15625 & 956 & 103823 & & & \\
\hline 64 & 31 & 16129 & 374 & 109561 & & & \\
\hline 81 & & 16384 & 689 & 113 & 601 & & \\
\hline 121 & & 16 & & & & & \\
\hline 12 & & 17161 & & & & & \\
\hline 128 & & 18769 & & 121801 & 31 & & \\
\hline 169 & & 19321 & & & & & \\
\hline 243 & & 19683 & & 128881 & & & \\
\hline 256 & & 22201 & & & & & \\
\hline 289 & & 22801 & & & & & \\
\hline 343 & & 24389 & & & & & \\
\hline 263 & & 24649 & & & & & \\
\hline 512 & & 26569 & & & & & \\
\hline 529 & & 27889 & & & & & \\
\hline 625 & & 28561 & & 148 & & & \\
\hline 729 & & 29791 & & & & & \\
\hline 841 & & 29929 & & & & & \\
\hline 961 & & 32041 & & & & & \\
\hline $2 L$ & & 32761 & & & 464 & & \\
\hline & & & & & & & \\
\hline & & 36481 & & & & & \\
\hline 81 & & 37249 & & & & & \\
\hline 48 & & 388 & & 17 & & & \\
\hline & & & & & & & \\
\hline & & 44 & & & & & \\
\hline & & 49729 & & & & & \\
\hline & & & & & & & \\
\hline & & & & & & & \\
\hline & & 52 & & & & & \\
\hline & & & & & & & \\
\hline & & & & & & & \\
\hline & & & & & & & \\
\hline & & & & & & & \\
\hline & & & & & & & \\
\hline & & & & & & & \\
\hline & & & & & & & \\
\hline & & & & 241081 & 120 & & \\
\hline & & 691 & & & & & \\
\hline & 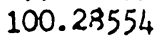 & & & & & & \\
\hline & & & & 31 & & & \\
\hline & & & & 262144 & 584.72 & & \\
\hline & & & & & & & \\
\hline & & & & 27 & & & \\
\hline & & & & & & & \\
\hline
\end{tabular}


TABLE I (continued)

\begin{tabular}{|c|c|c|c|c|c|}
\hline & $\Psi(x)-\theta(x)$ & & 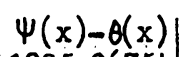 & & $\Psi(x)-\theta(x)$ \\
\hline & 907.56592 & & 1235.36754 & 2093809 & 77332 \\
\hline & 14.27887 & 1229881 & 1242.37875 & 2097152 & 1576.46646 \\
\hline & 668 & 1247689 & 1249.39715 & 2105401 & 7 \\
\hline & 690 & 126112 & 1256.42091 & 2111209 & .02786 \\
\hline 703921 & 934.44911 & 1274641 & 1263.45000 & 2128681 & 31336 \\
\hline 704969 & 938.93774 & 1295029 & 1268.14135 & 2163841 & 706 \\
\hline 707281 & 942.30504 & 1324801 & 1275.18973 & 2193361 & 90753 \\
\hline 727609 & 5380 & 1329409 & 1282.23986 & 219928 & 162 \\
\hline 734449 & 955.80724 & 1352569 & 1289.29861 & 2211169 & 1627.51387 \\
\hline 737881 & 962.56301 & 1371241 & 1296.36423 & 2217121 & 81973 \\
\hline 744769 & 969.32342 & 1394761 & 1303.43834 & 2229049 & \\
\hline 769129 & 976.09993 & 1408969 & 1310.51753 & 2247001 & 1649.44083 \\
\hline 776161 & 982. & 1419857 & 1313.35074 & 2248091 & 1654.31603 \\
\hline 779689 & 989.66431 & 1423249 & 1320.43497 & 2283121 & 63655 \\
\hline & 996 & 1442401 & 1327. & & .964 \\
\hline 822649 & 1003.26230 & & 1332.25327 & 2343961 & 1676.29867 \\
\hline 823543 & 1005.20821 & 369 & 1339.35412 & 2380849 & 64015 \\
\hline 829921 & 1012.02275 & 1481089 & 134 & 2399401 & 16 \\
\hline 844561 & 1018.84604 & 1495729 & 135 & 24 & \\
\hline 863041 & 1025.68015 & 1510441 & 1360 & 2430481 & 6852.6 \\
\hline 877969 & 1032.52283 & 361 & 1367 & 2455489 & 04218 \\
\hline 885481 & 1039. & 1530169 & 1374 & 2468041 & 172 \\
\hline 896809 & 1046 & 1560001 & 1382 & 2476099 & 1723.34 \\
\hline 908209 & 1053. & & 138 & 2493241 & 1730.71063 \\
\hline & 1057 & 23 & 139 & 2505889 & 1738.07771 \\
\hline 923521 & 1061 & 0729 & 1397.43636 & 2550409 & 174 \\
\hline 935089 & 1067 & 16 & 1404 & 2563201 & 175 \\
\hline & 1074 & & 715 & 353 & 175 \\
\hline & 1081 & 16 & 877 & 2582449 & 176 \\
\hline & 1088.6 & 16 & 1426. & 2588881 & 1772 \\
\hline & 109 & 16 & 1433.23975 & 2601769 & 177 \\
\hline 9 & 1102 & & 1054 & 161 & \\
\hline 1 & 110 & 16 & 1447 & 262 & 1794. \\
\hline & 1116 & 1708249 & 1454 & 264 & 1802.07315 \\
\hline & 112 & 1 & 146 & 2679769 & 47872 \\
\hline & 112 & & 14 & & 1814 \\
\hline 11 & 1131 & 17 & 1476 & 2745649 & 1821.8 \\
\hline & 1135 & & 147 & 2765569 & 182 \\
\hline & 114 & & & & 18 \\
\hline & 1149 & & 149 & 27 & 1844.03115 \\
\hline & 115 & & & 2825761 & 1847.79472 \\
\hline & 116 & & & 2866249 & \\
\hline & 1167 & & & 287 & 10606656 \\
\hline 601 & 1174.79 & 19531 & 1512.82992 & 2886601 & 1870.10339 \\
\hline & 1181 & & & 2920681 & 1877.5470 \\
\hline & 1188.73140 & 1985 & 1527. & 2961841 & 1884.99772 \\
\hline & 1195 & 20 & 1534 & 296372 & 1892.44954 \\
\hline $11 \varepsilon$ & 1202.69705 & & 1541.84792 & & \\
\hline & 1209 & & & & \\
\hline & 1216 & & & & \\
\hline & 1223 & 203348 & 156 & & \\
\hline & & & & & \\
\hline
\end{tabular}


TABLE II

$$
\lambda^{-} x<\psi(x)<\lambda^{+} x \text { if } n \leqslant x \leqslant 10^{8}
$$

\begin{tabular}{r|c|c||r|r|c}
\multicolumn{1}{r|}{$\mathrm{n}$} & $\lambda^{-}$ & $\lambda^{+}$ & \multicolumn{1}{c|}{$\mathrm{n}$} & \multicolumn{1}{c}{$\lambda^{-}$} & $\lambda^{+}$ \\
23 & 0.86583 & 1.03883 & 19379 & 0.99547 & 1.00458 \\
41 & 0.90602 & 1.03883 & 19423 & 0.99643 & 1.00458 \\
59 & 0.92237 & 1.03883 & 24281 & 0.99643 & 1.00361 \\
101 & 0.94842 & 1.03383 & 24297 & 0.99643 & 1.00297 \\
114 & 0.94842 & 1.03591 & 32059 & 0.99703 & 1.00297 \\
201 & 0.9481 .2 & 1.02728 & 32321 & 0.99770 & 1.00297 \\
227 & 0.96764 & 1.02728 & 43068 & 0.99770 & 1.00291 \\
285 & 0.96764 & 1.02117 & 59851 & 0.99770 & 1.00237 \\
347 & 0.97494 & 1.02117 & 60356 & 0.99770 & 1.00182 \\
469 & 0.97494 & 1.01802 & 60976 & 0.99770 & 1.00157 \\
569 & 0.97870 & 1.01802 & 69997 & 0.99787 & 1.00157 \\
664 & 0.97870 & 1.01386 & 70843 & 0.99816 & 1.00157 \\
684 & 0.97870 & 1.01364 & 88307 & 0.99851 & 1.00157 \\
1429 & 0.98708 & 1.01364 & 96020 & 0.99851 & 1.00153 \\
1630 & 0.98708 & 1.01196 & 102688 & 0.99851 & 1.00144 \\
1670 & 0.98708 & 1.00990 & 155941 & 0.99851 & 1.00121 \\
2657 & 0.98828 & 1.00990 & 175939 & 0.99870 & 1.00121 \\
2868 & 0.98828 & 1.00744 & 230481 & 0.99870 & 1.00115 \\
2974 & 0.98828 & 1.00662 & 303283 & 0.99891 & 1.00115 \\
3299 & 0.99002 & 1.00662 & 312229 & 0.99906 & 1.00115 \\
3461 & 0.99227 & 1.00662 & 356184 & 0.99906 & 1.00089 \\
3511 & 0.99237 & 1.00662 & 359808 & 0.99906 & 1.00079 \\
3948 & 0.99237 & 1.00649 & 445206 & 0.99906 & 1.00072 \\
5387 & 0.99330 & 1.00649 & 463447 & 0.99914 & 1.00072 \\
6385 & 0.99330 & 1.00640 & 467867 & 0.99938 & 1.00072 \\
6404 & 0.99330 & 1.00543 & 618736 & 0.99938 & 1.00057 \\
7045 & 0.99330 & 1.00517 & 1092893 & 0.97960 & 1.00057 \\
7459 & 0.99343 & 1.00517 & 1198538 & 0.99960 & 1.00049 \\
10359 & 0.99343 & 1.00458 & 1520786 & 0.99960 & 1.00037 \\
11801 & 0.99486 & 1.00458 & 1790309 & 0.99967 & 1.00037
\end{tabular}


Departamento de Matematica

Faculdade de Ciências de Lisboa

Lisbon, Portugal

1. K. I. Appel \& J. B. Rosser, Table for Estimating Functions of Primes, Comm. Res. Div. Tech. Rep. No. 4, Institute for Defense Analysis, Princeton, N.J., 1961.

2. D. N. Lehmer, List of Prime Numbers from 1 to 10006721, Carnegie Inst. of Washington, Publ. No. 165, Washington, 1914.

3. J. B. Rosser \& L. SChoenfeld, “Approximate formulas for some functions of prime numbers," Illinois J. Math., v. 6, 1962, pp. 64-94.

4. J. B. Rosser \& L. Schoenfeld, "Sharper bounds for the Chebyshev functions $\psi(x)$ and $\theta(x)$," Math. Comp., v. 29, 1975, pp. 243-269.

5. L. SCHOENFELD, "Sharper bounds for the Chebyshev functions $\psi(x)$ and $\theta(x)$. II," Math. Comp., v. 30, 1976, pp. 337-360. 